\title{
Smell and taste disorders during COVID-19 outbreak: a cross-sectional study on 355 patients.
}

\author{
Valeria Dell'Era, $\mathrm{MD}^{1}$ \\ ${ }^{1}$ University of Eastern Piedmont
}

May 12, 2020

\begin{abstract}
Background: As reported by increasing literature, a significant number of patients with SARS-CoV-2 infection developed smell/taste disorders.

Aim of this study is to determine prevalence and severity of these symptoms among laboratory confirmed SARS-CoV-2 patients. Secondary objective is to determine their onset/recovery time.

Methods: This cross-sectional study was conducted from March 10th to March 30th 2020 at Novara University Hospital during the COVID-19 Italian outbreak. The 355 enrolled patients answered a questionnaire at 14th (or more) days after proven infection.

Results: The overall population prevalence of both smell/taste or one of the two disorders was $70 \%$. They were first symptoms in $31(8,7 \%)$ patients.

Most patients reported a complete loss that in half of the cases (49.5\%) was fully recovered after 14 days, with a median recovery time of 10 days.

Conclusion: This study confirms high prevalence of smell/taste disorders in COVID-19 infection with self-recovery for half cases after about two weeks.
\end{abstract}

Valeria Dell'Era, MD (1), Filippo Farri, MD (1), Giacomo Garzaro, MD (2), Miriam Gatto, MD (3), Paolo Aluffi Valletti, MD (1), Massimiliano Garzaro, MD (1)

Affiliation

1 - ENT Division, University of Eastern Piedmont, Novara, Italy

2 - Department of Public Health and Pediatrics - University of Turin

3 - Division of Pediatrics, University of Eastern Piedmont, Novara, Italy

Acknowledgment

All authors equal contribute to this paper.

Authors declare $N O$ conflicts of interest, including relevant financial interests, activities, relationships, and affiliations.

Authors declare $N O$ financial and material support for the research.

We would like to thank you Dr. Fabio Carfagna for advice on the Manuscript.

Corresponding Author

Valeria Dell'Era 
ENT Division, University of Eastern Piedmont, 28100 Novara, Italy

valeria.dellera@gmail.com

Phone +3903213733403

ORCID ID: https://orcid.org/0000-0002-2171-4120

Running title: COVID-19 and smell

Key words: COVID-19, smell, taste, coronavirus, SARS - CoV-2 symptoms

ABSTRACT

Background: As reported by increasing literature, a significant number of patients with SARS-CoV-2 infection developed smell/taste disorders.

Aim of this study is to determine prevalence and severity of these symptoms among laboratory confirmed SARS-CoV-2 patients. Secondary objective is to determine their onset/recovery time.

Methods: This cross-sectional study was conducted from March 10th to March 30th 2020 at Novara University Hospital during the COVID-19 Italian outbreak. The 355 enrolled patients answered a questionnaire at $14^{\text {th }}$ (or more) days after proven infection.

Results: The overall population prevalence of both smell/taste or one of the two disorders was $70 \%$. They were first symptoms in $31(8,7 \%)$ patients.

Most patients reported a complete loss that in half of the cases (49.5\%) was fully recovered after 14 days, with a median recovery time of 10 days.

Conclusion: This study confirms high prevalence of smell/taste disorders in COVID-19 infection with self-recovery for half cases after about two weeks.

TEXT

\section{Introduction}

Anosmia, and hyposmia, the inability to perceive smells and a reduced or altered ability respectively, as well as taste perception alterations, are occasionally associated with respiratory virus infection, including the well known family of coronavirus ${ }^{1}$. Since mid-February 2020, SARS-CoV-2, a novel coronavirus initially identified in late 2019 in China, ${ }^{2}$ spread worldwide. As the infection was better characterized, main symptoms of fever, cough, tiredness and difficulty breathing were well characterized ${ }^{3}$. However several anecdotal case reports, ${ }^{4,5}$ case series, ${ }^{6-8}$ and surveys ${ }^{9-13}$ suggested that a significant amount of patients with suspected Sars-CoV-2 infection also developed anosmia/hyposmia and/or taste alteration, and in a relevant amount of patients, these were the first symptoms of COVID-19. Most of these studies, however, base their results on presumptive or symptoms based COVID-19 diagnosis. Only one study to date has assessed a population of laboratory confirmed patients, performing quantitative smell assessment, but without investigating taste. ${ }^{14}$

Limited knowledge is available on prevalence, severity and recovery time of these disorders in SARS-CoV-2 patients.

The aim of this study was to investigate the prevalence and severity of smell and taste disorders in a population of laboratory confirmed SARS-CoV-2 patients. A secondary objective was to investigate the onset and recovery time from the same symptoms.

\section{Materials and methods}

This is a cross-sectional study conducted from March 10th 2020 to March 30th 2020 at the ENT clinic of the University of Eastern Piedmont, Ospedale Maggiore della Carità in Novara, Italy. The study was carried out in accordance with the ethical standards of the Helsinki Declaration and was approved by the local ethical review board (Comitato Etico Interaziendale n.88/2020). 
The study included a convenience sample of SARS-CoV-2 polymerase chain reaction (PCR) confirmed cases being admitted for care or assessed and discharged during the study period.

Patients with confirmed SARS-CoV-2 infection were prospectively identified and recorded.

Administrative data were extracted from hospital databases and patients were offered a follow-up interview. Patients were informed about the study and asked to participate at the time of the follow-up interview (= or $>14$ days from diagnosis). Interviews were carried out by two attending physicians either in person or by phone using a structured questionnaire. Inclusion criteria were: Age $>18$-year old, informed consent and laboratory proven SARS-CoV-2 infection with a mild or moderate presentation of COVID-19 according to the severity classification of the Massachusetts General Hospital COVID-19 treatment guidance. ${ }^{15}$

Exclusion criteria were: declined informed consent, severe form of COVID-19.

Interview data was anonymously collected and no reward was offered for participation.

The questionnaire consisted of 22 questions, including general demographic information (age, gender), medical history (comorbidities, risks factors), SARS-CoV-2 related questions (risk exposure for occupational/family reasons, duration of infection, symptoms - fever, cough, dyspnea, fatigue or muscle pain, diarrhea or nausea) and specific information about the sense of smell and about taste (presence/absence, 0 to 10 numeric rating scale (NRS) of smell at baseline, during infection, after 14 days and at answering time - were 0 is "no sense of smell/taste" and 10 is "normal sense of smell/taste", time of onset/recovery). The survey is available in the supplementary online material. A clinically significant change in smell of taste perception was defined as having a score difference equal or greater than two points between study times.

\section{Statistical Analysis}

Smell and taste assessment were reported using data from the scales which have been treated as quantitative real values. Categorical variables were reported as a proportion while quantitative variables as median and ([25-75 quartile] range) where appropriate. Comparison of the baseline, worst and 14-days smell and taste perception was performed using Friedman's test. All statistical analysis has been performed using R 3.6.3, [R Core Team (2020). R: A language and environment for statistical computing. R Foundation for Statistical Computing, Vienna, Austria.] A p value $<0.05$ was considered significant.

\section{Results}

We interviewed a total of 355 patients with laboratory confirmed diagnosis of SARS-CoV-2. The population had a median time from symptoms onset of 23 ([17-28] range 2-53) days. Median age was 50 years old, 192 (54\%) were male. The overall population prevalence of both smell and taste or either one of the two disorders was $70 \%$ (249 out of 355). Among the two study symptoms $14(3.9 \%)$ patients presented a smell disorder with no alteration of taste, while $12(3.4 \%)$ patients presented with a taste disorder with no alternation of smell (but presented other SARS-CoV-2 systemic symptoms). Two patients presented with smell disorders as the sole SARS-CoV-2 symptom, while none had taste disorders as their only symptom. Smell or taste disorders were the first symptom of presentation in 31 (8.7\% of entire population and $13.0 \%)$ patients. Patients characteristics of the interviewed population, and for those presenting with olfactory and taste dysfunctions are presented in Table 1 .

\section{Smell Disorders}

$237(66 \%)$ patients reported a change in smell perception greater than two points between their baseline smell and the worst smell experienced during the infection. Of these, 128 (54\%) reported the smell dysfunction already present at the time of diagnosis.

These patients had a baseline smell perception of 10 ([9-10] range 3-10) with a worst smell perception during the infection of 0 ([0-2] range 0-7). At fourteen days from infection 115 (49.5\%) of patients declared a full recovery with smell as good as at baseline, while the remaining 122 declared a certain degree of remaining deficit with an overall population perception of 8 ([6-10] range 0-10). Variation of smell perception at different 
study period is presented in Figure 1A. 149 (62.9\%) patients reported full recovery at the time of interview, with a median recovery time of 10 ([7-15] range 1-25) days. No patient reported taking any specific therapy for their disorder.

Taste Disorders

$232(65.4 \%)$ patients reported a change in taste perception greater than two points between their baseline taste and the worst test experienced during the infection. These patients had a baseline taste perception of 10 ([9-10] range 5-10) with a worst taste perception during the infection of 0 ([0-2] range 0-10). At fourteen days from infection 117 (50.4\%) of patients declared a full recovery with test as good as at baseline while the remaining $115(49.6 \%)$ declared a certain degree of remaining deficit with an overall population perception of 8 ([6-10] range 0-10). Variation of taste perception at different study period is presented in Figure 1B.148 $(63.8 \%)$ patients reported full recovery at the time of interview, with a median recovery time of 10 ([7-15] range 2-25) days. No patient reported taking any specific therapy for their taste disorder.

Figure 2 shows the daily rate of recovery for both smell and taste disorders.

\section{Discussion}

Over the last weeks, an increasing number of Authors reported a high rate of olfactory and gustatory dysfunctions in COVID-19 patients, sometimes presenting as the first symptom. ${ }^{4-14}$

The main goal of the present study was to evaluate the prevalence of smell/taste disorders in a large COVID19 positive population, composed of patients who were either asymptomatic or presenting mild or moderate symptoms.

Our findings (66 and $65.4 \%$ respectively, without gender differences) are similar to those of Yan et al. about a smaller sample of 59 positive patients; ${ }^{10}$ a recent European multicenter study reported higher percentages both for smell and taste disorders, with a female prevalence ${ }^{12}$.

Since only one study by Mao et al. referred to the prevalence of chemosensory impairment in Asiatic COVID19 population $(5 \%)^{9}$, our data seem to corroborate the hypothesis by Lechien et al. that western positive patients (USA and Europe) may be more subject to the development of olfactory and gustatory disorders ${ }^{12}$.

The majority of patients in this study complained of complete anosmia/ageusia, confirming the observation that COVID-19 related impairment tends to induce a severe olfactory and gustatory dysfunction. ${ }^{10,12}$

Of particular interest is the analysis of the timing of anosmia: the loss of smell was noted in 54\% prior to diagnosis and appeared as the first symptom in $13 \%$ of cases. Recent onset of olfactory disorders may drive the physicians to treat these patients as possible COVID-19 positive: adequate precautions for the healthcare practitioners, PCR-testing and home self-isolation are crucial measures to avoid that such patients continuing to accidentally act as vectors of the disease.

Another pivotal issue of the research regards the recovery of olfactory and gustatory functions: $49.5 \%$ of patients reported a full regaining of both senses after 14 days since the beginning of the symptoms and this percentage improved to $62.9 \%$ at time of interview (23 days median, range 15-31), with a median recovery time of 10 days. Obviously, even if our follow- up is longer than other reports, ${ }^{10-12}$ it remains too short to give a conclusive interpretation regarding the prognosis.

Although the exact mechanism with which SARS-CoV-2 could impair smell and taste has not been definitively determined, two hypotheses appear to be plausible: damage to the olfactory epithelium, due to cell expression of angiotensin converting enzyme 2 (ACE2) receptors which act as a binding point for the virus ${ }^{16}$, or a direct assault on the olfactory neurons ${ }^{17}$. The relatively rapid and spontaneous recovery of most patients in the current and in other studies, tends to suggest an epithelial based pathogenesis, given the capability for the epithelium to quickly restore its functions after damage. ${ }^{10-12}$

According to some Authors, the presence of 15 variants of the ACE2 gene explains many ACE2 polymorphisms and differences in expression between European and Asian populations; this finding could be a cue 
to investigate if different patterns may influence susceptibility and clinical features of COVID-19 infection. ${ }^{18}$

The current study presents some limitations, shared by all similar researches. First of all, the patients did not undergo nasal endoscopy, specific imaging or objective smell assessment; these examinations would contribute to a better understanding of pathogenetic mechanisms and to define some prognostic factors about the functional recovery.

Second, the evaluation tool was a self-reported smell and taste score: It has been previously demonstrated that subjective evaluation of sense of smell is quite specific but less sensitive than objective testing. ${ }^{19}$ Moreover, the survey was rapidly developed by the Authors with the aim of collecting and sharing the most possible data in the shortest time; after such emergency contingency, additional validation is needed.

Third, even if our population is quite numerous, the sampling time was short and limited to a single center; future studies on larger samples can help to clarify the prevalence of anosmia/ageusia in COVID-19 patients.

Lastly, no patients in this study received specific treatment for olfactory or gustatory loss; currently no medical treatment has proved to be highly effective in post-viral anosmia. ${ }^{20-22}$ The potential risks of immunosuppression with the use of oral and some topical steroids also need to be borne in mind, consequently we agree with most authors, who recommend against specific therapy. ${ }^{4-8,12,13}$

A different approach could be applied to those patients with persistent olfactory disorders after complete recovery from COVID-19 and an exhaustive smell/taste assessment; in any case, further studies are needed in order to investigate potential benefits of treatment in selected cases.

Beyond the limitation, this is one of the first studies to provide the prevalence, the onset time, the severity and the recovery time of COVID-19 related smell and taste disorders; the follow-up time is acceptable and the sample is various and quite representative: Novara, indeed, is a small city (100000 inhabitants) near the border with the Lombardy Region, the epicenter of the Italian COVID-19 outbreak; at accrual time March, the $30^{\text {th }}$ - the positive patients in the town were almost 800 , of which 355 were enrolled in the current research.

The current study strongly confirms the high prevalence of olfactory and gustatory disorders in COVID-19 infection. ${ }^{22,23}$ Smell and taste loss may be used as indicators of potential contagion, and early identification may help to reduce the risk of spread, especially by paucisymptomatic cases.

\section{REFERENCES}

1. Suzuki M, Saito K, Min WP, et al. Identification of viruses in patients with postviral olfactory dysfunction. Laryngoscope. 2007;117(2):272-277. doi:10.1097/01.mlg.0000249922.37381.1e 2. Wu Z, McGoogan JM. Characteristics of and Important Lessons From the Coronavirus Disease 2019 (COVID-19) Outbreak in China: Summary of a Report of 72314 Cases From the Chinese Center for Disease Control and Prevention [published online ahead of print, 2020 Feb 24]. JAMA. 2020;10.1001/jama.2020.2648. doi:10.1001/jama.2020.2648 3. Huang C, Wang Y, Li X, et al. Clinical features of patients infected with 2019 novel coronavirus in Wuhan, China [published correction appears in Lancet. 2020 Jan 30;:]. Lancet. 2020;395(10223):497-506. doi:10.1016/S0140-6736(20)30183-5 4. Gautier JF, Ravussin Y. A New Symptom of COVID-19: Loss of Taste and Smell [published online ahead of print, 2020 Apr 1]. Obesity (Silver Spring). 2020;10.1002/oby.22809. doi:10.1002/oby.22809 5. Eliezer M, Hautefort C, Hamel AL, et al. Sudden and Complete Olfactory Loss Function as a Possible Symptom of COVID-19 [published online ahead of print, 2020 Apr 8]. JAMA Otolaryngol Head Neck Surg. 2020;10.1001/jamaoto.2020.0832. doi:10.1001/jamaoto.2020.0832 6. Gane SB, Kelly C, Hopkins C. Isolated sudden onset anosmia in COVID-19 infection. A novel syndrome? [published online ahead of print, 2020 Apr 2]. Rhinology. 2020;10.4193/Rhin20.114. doi:10.4193/Rhin20.114 7. Vaira LA, Salzano G, Deiana G, De Riu G. Anosmia and ageusia: common findings in COVID-19 patients [published online ahead of print, 2020 Apr 1]. Laryngoscope. 2020;10.1002/lary.28692. doi:10.1002/lary.28692 8. Giacomelli A, Pezzati L, Conti F, et al. Self-reported olfactory and taste disorders in SARS-CoV-2 patients: a cross-sectional study [published online ahead of print, 2020 Mar 26]. Clin Infect Dis. 2020;ciaa330. 
doi:10.1093/cid/ciaa330 9. Mao L, Jin H, Wang M, et al. Neurologic Manifestations of Hospitalized Patients With Coronavirus Disease 2019 in Wuhan, China [published online ahead of print, 2020 Apr 10]. JAMA Neurol. 2020;10.1001/jamaneurol.2020.1127. doi:10.1001/jamaneurol.2020.1127 10. Yan CH, Faraji F, Prajapati DP, Boone CE, DeConde AS. Association of chemosensory dysfunction and Covid-19 in patients presenting with influenza-like symptoms [published online ahead of print, 2020 Apr 12]. Int Forum Allergy Rhinol. 2020;10.1002/alr.22579. doi:10.1002/alr.22579 11. Kaye R, Chang CWD, Kazahaya K, Brereton J and Denneny III JC. COVID-19 Anosmia Reporting Tool: Initial Findings. 2020. Accepted for publication by Otolaryngology-Head and Neck Surgery. 12. Lechien JR, Chiesa-Estomba CM, De Siati DR, et al. Olfactory and gustatory dysfunctions as a clinical presentation of mild-to-moderate forms of the coronavirus disease (COVID-19): a multicenter European study [published online ahead of print, 2020 Apr 6]. Eur Arch Otorhinolaryngol. 2020;10.1007/s00405-020-05965-1. doi:10.1007/s00405-020-05965-1 13. Hopkins C, Surda P, Kumar N. Presentation of new onset anosmia during the COVID-19 pandemic [published online ahead of print, 2020 Apr 11]. Rhinology. 2020;10.4193/Rhin20.116. doi:10.4193/Rhin20.116 14. Moein ST, Hashemian SMR, Mansourafshar B, Khorram-Tousi A, Tabarsi P, Doty RL. Smell dysfunction: a biomarker for COVID-19 [published online ahead of print, 2020 Apr 17]. Int Forum Allergy Rhinol. 2020;10.1002/alr.22587. doi:10.1002/alr.22587 15. Massachusetts General Hospital COVID-19 Treatment Guidance. https://www.massgeneral.org/assets/MGH/pdf/news/coronavirus/covid19_domID_treatmentGuide.pdf 2020 (Accessed March 21, 2020) 16. Brann D, Tsukahara T, Weinreb C, Logan DW, Datta SR. Non-Neural Expression of SARS-CoV-2 Entry Genes in the Olfactory Epithelium Suggests Mechanisms Underlying Anosmia in COVID-19 Patients. Neuroscience; 2020. 17. Baig AM, Khaleeq A, Ali U, Syeda H. Evidence of the COVID-19 Virus Targeting the CNS: Tissue Distribution, Host-Virus Interaction, and Proposed Neurotropic Mechanisms. ACS Chem Neurosci. 2020;11(7):995-998). 18. Cao Y, Li L, Feng $\mathrm{Z}$, et al. Comparative genetic analysis of the novel coronavirus (2019-nCoV/SARS-CoV-2) receptor ACE2 in different populations. Cell Discov. 2020;6:11. Published 2020 Feb 24. doi:10.1038/s41421-020-0147-1 19. Boesveldt S, Postma EM, Boak D, et al. Anosmia-A Clinical Review [published correction appears in Chem Senses. 2017 Sep 1;42(7):607]. Chem Senses. 2017;42(7):513-523. doi:10.1093/chemse/bjx025 20. Hummel T, Whitcroft KL, Andrews P, et al. Position paper on olfactory dysfunction. Rhinol Suppl. 2017;54(26):1-30. 21. Harless L, Liang J. Pharmacologic treatment for postviral olfactory dysfunction: a systematic review. Int Forum Allergy Rhinol. 2016;6(7):760-767. doi:10.1002/alr.21727 22. Soler ZM, Patel ZM, Turner JH, Holbrook EH. A primer on viral-associated olfactory loss in the era of COVID-19 [published online ahead of print, 2020 Apr 9]. Int Forum Allergy Rhinol. 2020;10.1002/alr.22578. doi:10.1002/alr.22578

\section{FIGURE LEGENDS}

Figure1

Smell (A) and taste (B) perception (score) before developing symptoms (baseline), at the highest intensity of symptoms (worst) and after two weeks from their onset (14 days).

Figure2

Daily rate of recovery for both smell (blue) and taste disorders (red).

Table 1 Characteristics of patients with SARS-CoV-2 infection included in the study

\begin{tabular}{llll}
\hline & Overall Population $(\mathbf{n = 3 5 5})$ & Olfactory Symptoms $(\mathbf{n = 2 3 7})$ & Taste Symptoms \\
\hline Prevalence (\%) & $70 \%$ & $66 \%$ & $65.4 \%$ \\
Age (years), median [IQR] & $50[40-59.5]$ & $49[40-60]$ & $51[51-60]$ \\
Female, n (\%) & $163(45.9)$ & $115(48.5)$ & $110(47.4)$ \\
Smoker, n (\%) & $48(13.5)$ & $32(13.5)$ & $32(13.8)$ \\
Specific Risk Factors, n (\%) & & & \\
Healthcare Provider & $174(49)$ & $109(52.7)$ & $101(43.5)$ \\
Close Contact & $174(49)$ & $125(46)$ & $120(51.7)$ \\
Comorbidities, n (\%) & & &
\end{tabular}




\begin{tabular}{llll}
\hline & Overall Population $(\mathbf{n}=\mathbf{3 5 5})$ & Olfactory Symptoms $(\mathbf{n = 2 3 7})$ & Taste Symptoms \\
\hline Cardiac & $42(11.8)$ & $31(8.7)$ & $30(12.9)$ \\
Respiratory & $40(11.3)$ & $30(8.5)$ & $30(12.9)$ \\
Allergic/Nasosinusal & $38(10.7)$ & $26(7.3)$ & $23(9.9)$ \\
Other Symptoms, n (\%) & & & \\
Fever & $256(72.1)$ & $181(76.4)$ & $181(78)$ \\
Cough & $170(47.9)$ & $126(53.2)$ & $125(53.9)$ \\
Fatigue & $143(40.3)$ & $104(43.9)$ & $95(40.9)$ \\
Dyspnea & $77(21.7)$ & $50(21.1)$ & $54(23.3)$ \\
Diarrhea & $70(19.7)$ & $47(19.8)$ & $48(20.7)$ \\
\hline
\end{tabular}

A

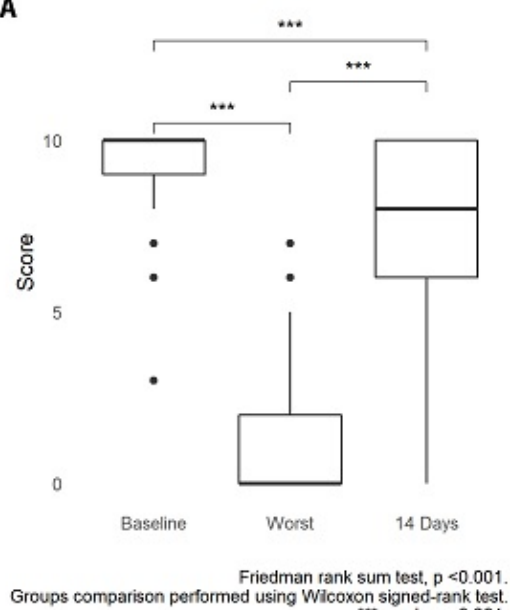

B

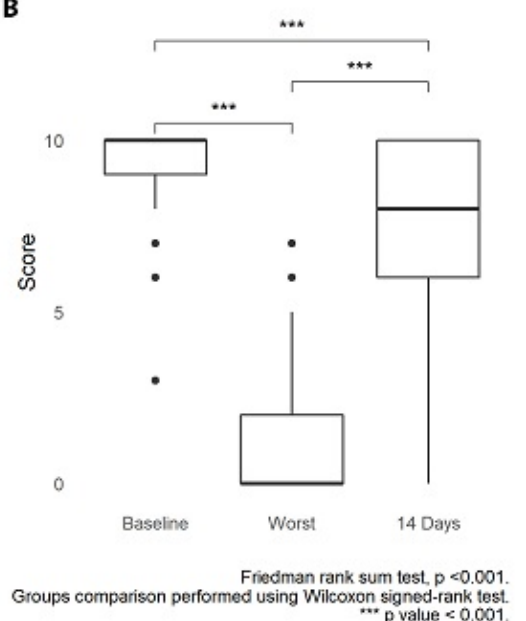

Figure 1: This is a caption

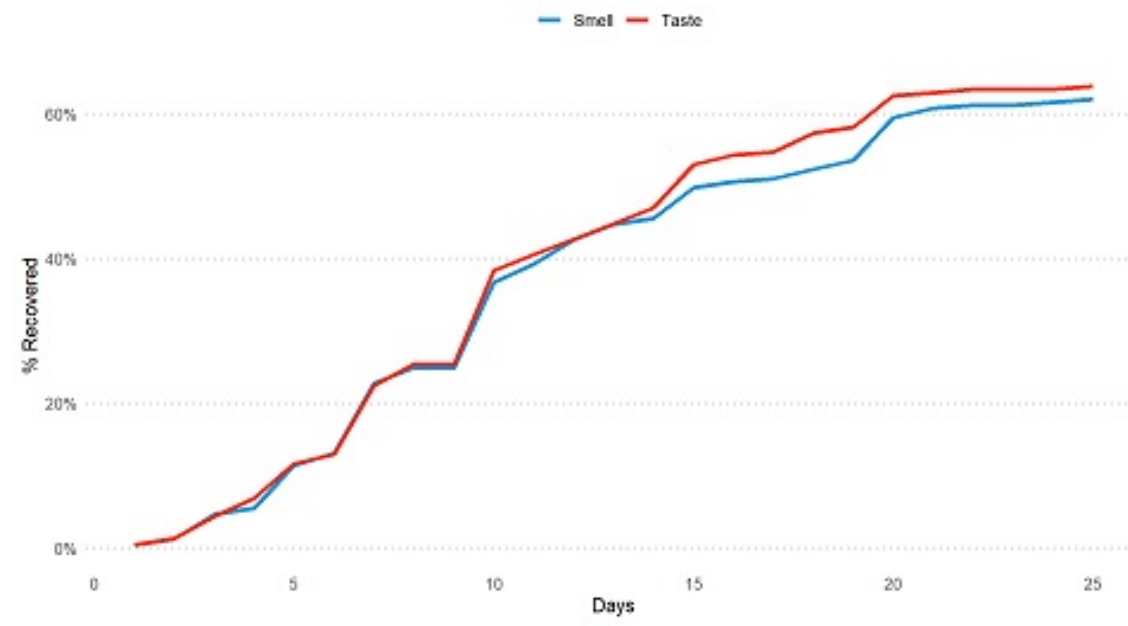

Figure 2: This is a caption 


\section{Hosted file}

Survey.docx available at https://authorea.com/users/312328/articles/450599-smell-and-tastedisorders-during-covid-19-outbreak-a-cross-sectional-study-on-355-patients 